\title{
Gestational breast cancer. Surgical treatment, pregnancy and fetal outcome
}

\author{
Olivia IONESCU ${ }^{1}$, Nicolae BACALBASA ${ }^{2}$ \\ 1"Fichtelgebirge" Hospital, Marktredwitz, Bavaria, Germany \\ 2"Carol Davila" University of Medicine and Pharmacy, Bucharest, Romania
}

\begin{abstract}
Background. Gestational breast cancer (GBC) is also known as pregnancy-associated breast cancer and it comprises all the breast cancers $(B C s)$ which are diagnosed either during pregnancy or in the first year after delivery or during the lactation period. At present it has been confirmed that the breast malignancies are the most common forms of cancer in pregnant women with a constant increase in its incidence because of the continuous postpone in childbearing especially in women older than 40 years. However, when diagnosed during the pregnancy, the treatment modalities of the $B C$ are complex and difficult to establish as it must be considered the impact of the treatment both on the child and the course of pregnancy.

Purpose. Using an online search on Pubmed, our aim was to make a review of the treatment possibilities of a pregnant woman presenting a breast malignant tumor. We have concentrated our paper on the surgical treatment and the possibility of an oncoplastic reconstruction types, the facts of radiotherapy during pregnancy and the prognosis of the GBC particularly in women who opt to continue the pregnancy. A resume of the epidemiology of $G B G$ is also presented.

Method. The following key words have been on Pubmed introduced: "breast cancer", "pregnancy", ,"staging", "chemotherapy" and "radiotherapy". As mentioned above, we have tried to select the BC cases diagnosed and treated during pregnancy for which the decision of the patient was to continue the pregnancy in spite of the diagnosis. We further aimed to present the prognosis of the pregnancy-associated $B C$, namely the pregnancy and fetal outcome, and to investigate if the decision to terminate the pregnancy is associated with a survival benefit.

Conclusion. The surgical treatment of pregnancy-associated $B C$ does not differ from that of non-pregnancy BC. Axillary LN-dissection is permitted while the data on the safety of sentinel-LN are still poor. Elective termination of the pregnancy has no impact on the overall survival of the patient.
\end{abstract}

Keywords: gestation, breast cancer, surgery

Abbreviations: $\mathrm{BC}=$ breast cancer; $\mathrm{LN}=$ lymph node; $\mathrm{BCS}=$ breast conservative surgery

\section{INTRODUCTION AND EPIDEMIOLOGY}

Although rare, breast cancer $(\mathrm{BC})$ remains the most frequent form of cancer that is associated with the gestational period - both in the prenatal and in the postnatal period - with an approximated incidence of 15 to $35 / 100,000$ deliveries (1). The number of $B C$ cases in the prenatal period are fewer than those diagnosed in the postnatal period (2). Moreover, it seems that incidence rate is higher in younger women, in particularly in women under 30 years, between $10 \%$ and $20 \%$ of patients being identified with $B C$ either during pregnancy or in the first postpartum year (3). Pregnant women who inherit either the BRCA 1 or BRCA 2 mutation may also consist a significant percentage among the gestational $\mathrm{BC}$ cases. Moreover, although there are no sufficient scientific evidences, the inheritance of the BRCA 2 mutation may represent 
an important risk for GBG even in case of multiparity which is generally considered to protect against $B C(4)$.

Some studies have reported an advanced stage of the $B C$ at the moment of diagnosis during pregnancy, namely between $65 \%$ and $90 \%$ stage II-III BCs compared to $45-66 \%$ stage II-III BCs in non-pregnant women $(5,6)$. These higher rates can be attributed to the hormonal changes that occur in the breast tissue during pregnancy such that the self-examination and mammography are associated with false-negative results $(3,7)$. Therefore, the identification of the BC takes places in an advanced stage, most frequently when the malignant cells have already spread into the regional lymph nodes (LNs). The hypothesis has been reported in the review conducted by Nettleton et al (8) whose research in the specialized literature has shown that the incidence of metastatic LNs increases with each 1-month delay in BC identification. Moreover, when considering the biology of the tumor, GBC appear to be more aggressive as results from the most common histologic and prognostic characteristics: more often they are moderate or low differentiated tumors (G2-G3), overexpression of the human epidermal growth factor receptor 2 , high rates of the nuclear antigen $\mathrm{Ki}-67 \%$ while the expression of the estrogen and progesterone receptors is absent $(3,9)$.

The treatment of GBG impacts the prognosis of the patients and should consider three important issues: the effect of the treatment - locally or systemic - on the fetus, the outcome of the delivery and the maternal health. These three issues will be discussed also in this review. With regard to the main theme of the review - the treatment of GBG - excepting that harms the fetus - the guidelines for GBG treatment are the same as those for non-pregnant women (10). The treatment should be initiated with a curative intent and should not be postponed due the presence of pregnancy. The elective termination of the pregnancy has been demonstrated to have no positive influence on the prognostic of the BC (11). Furthermore, similar to primary $B$ in non-pregnant women, before the treatment initiation, it is essentially to identify distant metastases taking with imagistic methods taking into account the need to protect the fetus. Staging of the disease in pregnancy can be made using abdomen sonography, chest radiography with fetal protection and skeletal magnetic resonance imaging without contrast (12).
Even though it has a dramatically impact on the women's psychic, information on the impact of the GBG and its staging modalities on the pregnancy, infant and delivery outcome should be detailed by the doctor. Moreover, every decision on the appropriate approach of the pregnant woman with $\mathrm{BC}$ should be discussed in a multidisciplinary tumor conference consisted of a surgeon, obstetrician and an oncologist. In this paper we have aimed to make a research in the literature in order to present the possible loco-regional and systemic treatment of BC identified during pregnancy in women who opt to give birth to the child. As mentioned above, we will also resume the available data on the prognosis of pregnancy-associated BC.

\section{SURGICALLY TREATMENT}

\section{Breast conservative surgery vs. mastectomy}

Similar to non-pregnant women the loco-regional treatment of GBG during pregnancy is surgically, respectively through breast conservative surgery, modified or total mastectomy with axillary LNs approach. It is generally accepted that the surgical treatment can be initiated any time in pregnancy without impairing the fetus (13). However, the data regarding the safety of the surgery in the first pregnancy trimester has been extrapolated from expert opinions, observational studies and trials during caesarean delivery (14).

Similar to the non-gestational BC, breast conservative surgery (BCS) is considered to be feasible with no significant effect on the recurrence rate and good prognostic outcomes both for the baby and the mother (15). Owing to the teratogenic effects of the radiation, the adjuvant radiotherapy of the ipsilateral breast in order to avoid recurrence should be initiated after delivery (16). Even though radical mastectomy has been considered the optimal treatment in pregnant women with clinical stages I or II as it does not require adjuvant radiotherapy, the available data, although limited, support and encourage the use of BCS showing that similar overall survival rates between women who underwent a radical mastectomy and women who received BCS (17-19).

With regard to the impact of BCS on the pregnancy evolution, BCS (and mastectomy) can be performed any time in the pregnancy also in the first trimester $(20,21)$. However, the doctor should be aware of the fact that a BCS 
performed during early gestation can be associated with a poor local control of the disease as maintaining the pregnancy implies a long delay in the initiation of adjuvant radiotherapy (22). In spite of the lack of information on the effects of delayed starting of radiotherapy after BCS, most of data being extracted from retrospective studies which have included non-pregnant women, Chen et al (23) showed that postponing the initiation of RT with 1 month results in an 1\% increased risk of developing local recurrences. Therefore, the decision to delay RT in women who opt for not interrupting the pregnancy after BCS performed in the first trimester and further adjuvant chemotherapy should be carefully weighted taking into consideration the risk associated with RT applied during pregnancy and the lack of precise information regarding the effects of a delayed RT on the local control of the disease. However, if adjuvant chemotherapy is necessary, it is administered after surgery and generally requires 6 months which means an automatic delay of RT that is expected to take place after delivery. In non-pregnant women, the initiation of RT in pregnant women after 6 month of chemotherapy has not been proven to impact the prognosis of the disease (24).

As mentioned above, pregnant women tend to present with an advanced stage of disease due to the changes in the density of the breast during pregnancy that prevent an earlier identification of the tumor. On one side, because of the size of the tumor at the initial presentation, on the other side, because of elimination of RT after mastectomy, a significant proportion of women decide themselves for a radical or modified mastectomy $(12,25)$. Similarly to BCS, the mastectomy can be performed any time during pregnancy, with special consideration to GBC in first trimester pregnancy that require radiotherapy even though the women opted for a mastectomy. Also in these cases, RT should be started after delivery as the fetal exposure to radiation during pregnancy is considered to be very high regardless the trimester of pregnancy (25).

\section{Oncoplastic reconstruction}

Oncoplastic reconstruction can be made immediately after the mastectomy or after delivery which means a delayed reconstruction. The major disadvantage of an immediate reconstruction represents the need to perform a reexcision or a mastectomy (for women who initially received lumpectomy) when the final histopathologic examination confirms the presence of positive margins of resection (26). On the other side, an immediate reconstruction is associated with an increased self-esteem, a positive psychic, emotional health and self-confidence when it comes to sexuality and body image (27). Taking into account all these benefits, Toesca and coworkers (24) propose an immediate reconstruction after mastectomy also in pregnant women which should preferentially be made with a tissue expander knowing that this technique usually lacks obstetrical complications and does not require long operating times (28). Other techniques such as ipsilateral definitive implant and contralateral reduction or reconstruction with autologous tissue are generally not recommended during pregnancy. The first technique is usually inadequate due to the modifications of the breast tissue during pregnancy and in the postpartum period while the second method is associated with an increased risk of peri- and postoperative complications (24).

The oncoplastic reconstruction procedures can be divided into: autologous procedures using tissue from abdomen, posterior thorax, abdominal thigh and buttock and procedures using prosthetic devices.

The autologous procedures can be:

- a) The volume displacement techniques refer to: mastopexy, reduction mammoplasty and adjacent tissue rearrangement. All of these methods aim to rearrange the form of the breast by removing the cavity where the breast tumor was placed taking care to maintain a viable nipple and areola complex. The reduction mammoplasty appears to be most used and involves a partial mastectomy followed by simultaneously performed ipsilateral and contralateral reduction mammoplasty. The cavity where the tumor was will be filled with parenchymal breast tissue. Generally, the volume displacement procedures are utilized for women with voluminous breasts (29).

- b) The volume replacement techniques refer to: latissimus dorsi flap or thoracodorsal artery perforator flap in case of defects in the superior, inferior and lateral part of the breast and internal mammary artery perforator flaps for medial defects. These procedures are also aimed at filling the tumor cavity using free tissue transfer or adjacent pedicle flaps and are proposed to 
women with small breasts for which a reduction mammoplasty cannot will be inadequate due to the insufficient breast tissue. Regarding the efficiency of different volume replacement disorders, a study conducted by Gendy and published in 2003 has shown that skin-sparing mastectomy, with latissimus dorsi flaps and oncoplastic surgery with latissimus dorsi flaps were associated with favourable impacts regarding aesthetic outcome although for the latter technique fewer side surgical and physical effects have been reported (30).

\section{Sentinel Node Biopsy in pregnancy}

As known, the management of the axilla is essential in the management of $\mathrm{BC}$ as it provides a good local control of the disease, brings information on the adjuvant systemic treatment and the patient's prognostic (31). Pregnant women with clinically positive axilla or with inflammatory breast disease should be offered a complete axillary LN dissection (32).

When it comes to the safety of the sentinel-LN biopsy, the data is controversial. The reports conducted by Gentillini $(33,34)$ proved that the concept of sentinel-LN during pregnancy is safe and should be applied in order to stage BC and establish whether a complete axillary dissection is necessary or not. He used 99mTc-labeled human albumin colloid particles (99mTc-HSA nanocolloids) in a volume of $0.2 \mathrm{ml}$ 16-18 $\mathrm{h}$ which has been injected peritumoral before the surgical intervention and demonstrated that lymphoscintigraphy can be successfully applied also in pregnant patients the risk of radiation being very low as well and a negligible risk to the fetus. The radioactive activity measured in blood and excreted in the urine 16 hours after injection was less than $2 \%$ which means very low - below the 50 mGy threshold absorbed dose for adverse effects.

Other reports $(35,36)$ have also confirmed a very low absorbed dose to the fetus or embryo which further suggest a harmless risk with respect to cardiac malformations or mental retardation. In order to further reduce the risk of embryo/fetus irradiation the radio colloid should be preferably injected on the day of operation (in the morning) to avoid a too long exposure to radiation.

When it comes to the substance that should be peritumoraly injected, the supravital dyes are forbidden. Isosulfan blue can cause allergic or anaphylactic maternal reactions which can negatively impact the fetus. This risk has been estimated to be approximately 1\%. Methylene blue is associated with theratogenic effects especially in the first trimester when it can lead to jejunal atresia (37).

In spite of the above presented favorable results, the safety and efficacy of lymphatic mapping in pregnant women remains controversial. There are no sufficient supporting studies to confirm that the injected radioisotope remains stocked at the site of the tumor and does not passage the placenta to the embryo/fetus. On the other hand, due to the physiological modifications of the breast during pregnancy, the lymphatic patterns can be transformed making the identification of the Sentinel-LN difficult (38). However, every clinically suspicious axillary LN should be evaluated using sonography and fine needle biopsy with aspiration for the histologic confirmation.

In the same way, external radiotherapy in $\mathrm{BC}$ remains contraindicated in pregnant women due the increased risk of organ malformations as well as the high probability of developing cancer during childhood following irradiation during pregnancy (39). The reported short-term fetal outcomes include: preterm birth, spontaneous abort, perinatal death, ventricular sept defects, undescended left testicle, loss of the sense of hearing and attention deficit (40). Therefore, owing to the side effects on child and due to the few data on the safety and efficacy of radiation during the first and second trimester of pregnancy, external beam radiation therapy remains forbidden in pregnant $B C$ patients while its necessity as well as the problem of delaying radiotherapy in women who receive operation in the first trimester should be considered for each patient individually and discussed in a multidisciplinary tumor conference (24).

\section{Prognosis and the decision to terminate the pregnancy}

Generally, pregnant-associated BC diagnosed in stages I and II has the same overall survival rate as non-associated BC $(41,42)$. However, it seems that an advanced age (for example pregnant women older than 35 years old) or an advanced stage at the moment of diagnosis or tumors diagnosed in the first postpartum year have a negative impact on the patient's prognosis in terms of overall survival as well as disease-free survival rate $(43,44)$. As expected, pregnant women with larger tumors present with axillary $\mathrm{LN}$ metastases and, considering 
the age of the patient, the tumor is more likely to not present the expression of the hormonal receptors which further worsens the prognosis $(44,45)$. Other studies showed that a longer period between the appearance of the first tumor symptom and the definitive (histologic) diagnosis through fine needle biopsy is associated with a reduced overall survival rate (46).

It must be emphasized that there is a significant difference in the survival outcome between pregnant women with $\mathrm{BC}$ and $\mathrm{BC}$ diagnosed in the first postpartum year. Pregnant women with $\mathrm{BC}$ have been proved to have the same progression-free and overall survival as non-pregnant women (11). Furthermore, the systemic therapy performed in the second and third trimester resulted in a better five-year and overall survival rate compared to non-pregnant women with the same age and stage of the tumor (47). The opposite situation was observed in the case of BCs diagnosed in the first postpartum year which has been associated with a higher mortality rate compared to non-associated $B C$ even though the stage an size of the tumor, the hormonal receptor and Her-2/neu status as well as the type of treatment performed were similar. $B C$ in the first postpartum year has a poorer overall survival rate than $B C$ during pregnancy, probably due to the differences in the therapeutic attitude between pregnant and non-pregnant women - for example, performing the taxane-based chemotherapy after delivery (49).

The prognosis of the fetus has also been reported, the majority of studies demonstrating that chemotherapy has no impact on the child's early development respectively on the cardiac - especially cardiac side effects (ventricular wall thickness, ejection fraction) after in utero exposure to anthracyclines - and neurologic development. The results showed similar outcome between children exposed to chemotherapy (mandatory after the first trimester) and children of non-cancer patients. Regarding the gestational age at the moment of delivery, the most frequent reported gestational age was 3537 weeks which means a late preterm delivery which can be owed to the decision to terminate the pregnancy for the further performance of the adjuvant treatment (49).

Between delivery and the chemotherapy cycle a period of 3 weeks is recommended in order to allow the excretion from the fetus of the chemotherapeutic drugs. This can be accomplished only by the term infants which implies the necessity to receive the last chemotherapy cycle before delivery at about 32-33 weeks of gestation. After the birth of the child, the chemotherapy or the adjuvant radiotherapy will be initiated on 7 or 8 day after delivery. Pregnant-associated BC does not consists an indication for Caesarean Section while the risk of a spontaneous preterm labor is similar to the pregnant women without BC (25).

The elective termination of pregnancy has not been proved to improve the maternal prognosis, on the contrary, a poorer overall survival rate has been observed in women who decide to interrupt the pregnancy. It is evident that the decision to terminate the pregnancy should be individually made and the patient has the right to be fully informed about the complications of the surgical and/or systemic treatment during pregnancy as well as the effects of the management (especially of the chemotherapy) on her fertility (50).

A dissemination of the malignant cells to the unborn through the placenta (a vertical transmission of the disease) could be possible but no cases of cancer in children who were in utero exposed to chemotherapy have been reported (51).

\section{CONCLUSIONS}

As stated previously, the current indications for pregnancy-associated $B C$ are similar to those which regard non-associated BC. The exception is the performance of chemotherapy that is contraindicated in the first trimester owing to the high risk of malformations, and radiotherapy, which currently is forbidden during pregnancy. The loco-regional treatment of GBG includes BCS or mastectomy, the decision to perform one or another being influenced by the tumor characteristics and the patient's preference. The concept of sentinel-LK cannot be implemented during pregnancy, but patients with a clinically positive axilla should receive an axillary LN-dissection. The immediate breast reconstruction should preferably be done using tissue expanders, a technique which is rapid and does not imply serious complications.

The elective termination of pregnancy does not improve the patient's prognosis while an infant born to women with BC has similar outcome to that of a child with the same gestational age.

Acknowledgement: This work was supported by a grant of the Romanian National Authority for Scientific Research and Innovation, CNCS - UEFISCDI, project number PN-II-RU-

TE-2014-4-2533. 


\section{REFERENCES}

1. Andersson T.M., Johansson A.L., Hsieh C.C. et al. Increasing incidence of pregnancy-associated breast cancer in Sweden. Obstet Gynecol 2009; 114:568.

2. Stensheim H., Møller B., van Dijk T., Fosså S.D. Cause-specific survival for women diagnosed with cancer during pregnancy or lactation: a registry-based cohort study. J Clin Oncol 2009; 27:45.

3. Ishida T., Yokoe T., Kasumi F. et al. Clinicopathologic characteristics and prognosis of breast cancer patients associated with pregnancy and lactation: analysis of case-control study in Japan. Jpn J Cancer Res 1992; 83:1143.

4. Cullinane C.A., Lubinski J., Neuhausen S.L. et al. Effect of pregnancy as a risk factor for breast cancer in BRCA1/ BRCA2 mutation carriers. Int J Cancer 2005; 117:988

5. Valentini A., Lubinski J., Byrski T. et al. on behalf of the Hereditary Breast Cancer Clinical Study Group. The impact of pregnancy on breast cancer survival in women who carry a BRCA1 or BRCA2 mutation. Breast Cancer Res Treat 2013; 142:177-85.

6. Smith L.H., Danielsen B., Allen M.E., Cress R. Cancer associated with obstetric delivery: results of linkage with the California cancer registry. Am J Obstet Gynecol 2003; 189:1128.

7. Bonnier P., Romain S., Dilhuydy J.M. et al. Influence of pregnancy on the outcome of breast cancer: a case-control study. Societe Francaise de Senologie et de Pathologie Mammaire Study Group. Int J Cancer 1997;72:720-7.

8. Nettleton J., Long J., Kuban D., Wu R., Shaefffer J., El-Mahdi A. Breast cancer during pregnancy: quantifying the risk of treatment delay. Obstet Gynecol 1996; 87:414-18.

9. Reed W., Hannisdal E., Skovlund E., Thoresen S., Lilleng P., Nesland J.M. Pregnancy and breast cancer: a populationbased study. Virchows Arch 2003; 443:44-50.

10. Azim H.A. Jr., Botteri E., Renne G. et al. The biological features and prognosis of breast cancer diagnosed during pregnancy: a case-control study. Acta Oncol. 2012; 51:653-661.

11. Amant F., von Minckwitz G., Han S.N. et al. Prognosis of women with primary breast cancer diagnosed during pregnancy: results from an international collaborative study. J Clin Oncol. 2013;31:2532-2539

12. Amant F., Deckers S., Van Calsteren K. et al. Breast cancer in pregnancy: recommendations of an international consensus meeting. Eur J Cancer 2010; 46:3158.

13. ACOG Committee on Obstetric Practice. ACOG Committee Opinion No. 474: nonobstetric surgery during pregnancy. Obstet Gynecol 2011; 117:420.
14. Woo J.C., Yu T., Hurd T.C. Breast cancer in pregnancy: a literature review. Arch Surg 2003; 138:91.

15. Berry D.L., Theriault R.L., Holmes F.A. et al. Management of breast cancer during pregnancy using a standardized protocol. 1999. J Clin Oncol 17:855-861

16. Kal H.B., Struikmans H. Radiotherapy during pregnancy: fact and fiction. Lancet Oncol 2005; 6:328.

17. Gentilini O., Masullo M., Rotmensz N. et al. Breast cancer diagnosed during pregnancy and lactation: biological features and treatment options. 2005. Eur J Surg Oncol 31:232-236

18. Kuerer H.M., Gwyn K., Ames F.C., Theriault R.L. Conservative surgery and chemotherapy for breast carcinoma during pregnancy. Surgery 2002; 131:108.

19. Rodriguez A.O., Chew H., Cress R. et al. Evidence of poorer survival in pregnancyassociated breast cancer. Obstet Gynecol. 2008; 112(1): 71-78.

20. Gemignani M.L., Petrek J.A., Borgen P.I. Breast cancer and pregnancy. Surg Clin North Am 1999; 79:1157.

21. Duncan P.G., Pope W.D.B., Cohen M.M., Greer N. Fetal risk of anesthesia and surgery during pregnancy. 1986. Anesthesiology 64:790-794

22. Hershman D.L., Wang X., McBride R., Jacobson J.S., Grann V.R., Neugut A.I. Delay in initiating adjuvant radiotheraphy following breast conservation and its impact on survival. 2006. Int J Radiat Oncol Biol Phys 65(5):1353-1360

23. Chen Z., King W., Pearcey R., Kerba M., Mackillop W.J. The relationship between waiting time for radiotherapy and outcome: a systematic review of the literature. 2008. Radiother Oncol 87:3-16

24. Toesca A., Gentilini O., Peccatori F., Azim Jr. H.A, Amant F. Locoregional treatment of breast cancer during pregnancy. Gynecol Surg. 2014; 11:279284.

25. Cardonick E. Pregnancy-associated breast cancer: optimal treatment options. International Journal of Women's Health 2014: 6 935-943.

26. Morrow M. et al. Surgeon recommendations and receipt of mastectomy for treatment of breast cancer. 2009. JAMA 14:1551-1556

27. Fernandez-Delgado J., Lopez-Pedraza M.J., Blasco J.A. et al. Satisfaction with and psychological impact of immediate and deferred breast reconstruction. Ann Oncol. 2008. 19: 1430-1434

28. Lohsiriwat V., Peccatori F.A., Martella S., Azim H.A. Jr., Sarno M.A., Galimberti V., DeLorenzi F., Intra M., Sangalli C., Rotmensz N., Pruneri G., Renne G., Schorr M.C., Nevola Teixeira L.F., Rietjens M., Giroda M., Gentilini O. Immediate breast reconstruction with expander in pregnant breast cancer patients. Breast 22(5): 657-660

29. Losken A, Hamdi M. Partial breast reconstruction: current perspectives. Plast Reconstr Surg 2009; 124:722.

30. Gendy R.K., Able J.A., Rainsbury R.M. Impact of skin-sparing mastectomy with immediate reconstruction and breastsparing reconstruction with miniflaps on the outcomes of oncoplastic breast surgery. Br J Surg 2003; 90:433.

31. Lyman G.H., Giuliano A.E., Somerfield M.R. et al. American Society of Clinical Oncology guideline recommendations for sentinel lymph node biopsy in early-stage breast cancer. $\mathrm{J}$ Clin Oncol 2005; 23:7703.

32. Schwartz G.F., Giuliano A.E., Veronesi U. Consensus Conference Committee. Proceedings of the consensus conference on the role of sentinel lymph node biopsy in carcinoma of the breast, April 19-22, 2001, Philadelphia, Pennsylvania. Cancer 2002; 94: 2542.

33. Gentilini O., Cremonesi M., Trifiro G., et al. Safety of sentinel node biopsy in pregnant patients with breast cancer. Ann Oncol. 2004; 15(9): 1348-1351

34. Gentilini O., Cremonesi M., Toesca A. et al. Sentinel lymph node biopsy in pregnant patients with breast cancer. Eur J Nucl Med Mol Imaging; 2010; 37(1): 78-8.

35. Russell J.R., Stabin M.G., Sparks R.B., Watson E. Radiation absorbed dose to the embryo/fetus from radiopharmaceuticals. 1997. Health Phys 73:756-769

36. Russell J.R., Stabin M.G., Sparks R.B. Placental transfer of radiopharmaceuticals and dosimetryin pregnancy. Health Phys 73:747-755

37. Khera S.Y., Kiluk J.V., Hasson D.M. et al. Pregnancy-associated breast cancer patients can safely undergo lymphatic mapping. 2008. Breast J 14(3): 250-254

38. Keleher A., Wendt R. 3rd, Delpassand E. et al. The safety of lymphatic mapping in pregnant breast cancer patients using Tc-99m sulfur colloid. Breast $J$ 2004; 10:492.

39. Mazonakis M., Varveris H., Damilakis Jetal. Radiationdoseto conceptus resulting from tangential breast irradiation. 2003. Int J Radiat Oncol Biol Phys 55(2):386-391

40. Luis S.A., Christie D.R., Kaminski A. et al. Pregnancy and radiotherapy: management options for minimising risk, case series and comprehensive literature review. 2009. J Med Imaging Radiat Oncol 53(6): 559-568

41. Halaska M.J., Pentheroudakis G., Strnad P. et al. Presentation, management and outcome of 32 patients with pregnancyassociated breast cancer: a matched controlled study. Breast J. 2009; 15(5):461-467

42. Smith L.H., Danielsen B., Allen M.E., Cress R. Cancer associated with obstetric 
delivery: results of linkage with the California cancer registry. Am J Obstet Gynecol. 2003; 189(4):1128-1135.

43. Ibrahim E.M. Ezzat A.A., Baloush A., Hussain Z.H., Mohammed G.H.

Pregnancy-associated breast cancer: a case-control study in a young population with a high-fertility rate. Med Oncol. 2000; 17(4): 293-300.

44. Anderson B.O., Petrek J.A., Byrd D.R., Senie R.T., Borgen P.I. Pregnancy influences breast cancer stage at diagnosis in women 30 years of age and younger. Ann Surg Oncol. 1996; 3(2):204-211

45. Rodriguez A.O., Chew H., Cress R. et al. Evidence of poorer survival in pregnancy-

Conflict of interest: none declared associated breast cancer. Obstet Gynecol. 2008; 112(1):71-78.

46. Bonnier P., Romain S., Dilhuydy J.M. et al. Influence of pregnancy on the outcome of breast cancer: a case-control study. Societe Francaise de Senologie et de Pathologie Mammaire Study Group. Int J Cancer. 1997; 72(5):720-727.

47. Litton J.K., Warneke C.L., Hahn K.M. et al. Case control study of women treated with chemotherapy for breast cancer during pregnancy as compared with nonpregnant patients with breast cancer. Oncologist 2013; 18:369.

48. Azim H.A. Jr., Santoro L., Russell-Edu W. et al. Prognosis of pregnancy-associated breast cancer: a meta-analysis of 30 studies. Cancer Treat Rev 2012; 38:834.

49. Amant F., Vandenbroucke T., Verheecke M. et al. Pediatric Outcome after Maternal Cancer Diagnosed during Pregnancy. N Engl J Med 2015; 373:1824.

50. Psyrri A., Burtness B. Pregnancyassociated breast cancer. Cancer J. 2005;11(2):83-95.

51. Dessolle L., Dalmon C., Roche B., Daraï E. Placental metastases from maternal malignancies: review of the literature. $J$ Gynecol Obstet Biol Reprod (Paris) 2007; 36:344. 